\title{
COMPUTATIONAL SCREENING OF FLAVONOIDS FOR HCV INHIBITION
}

\author{
Pooja Karkera ${ }^{1}$, Sanceep Telkar ${ }^{2}$, Usha BM ${ }^{3}$, Kumara Swamy $\mathrm{HM}^{4}$ and Ravikumar Y $\mathbf{S}^{5}$ \\ ${ }^{I}$ Department of Biotechnology, M.S. Ramaiah Institute of Technology, Bangalore-560 054, Karnataka, India \\ ${ }^{2}$ Department of Biotechnology, Jnana Sahyadri Kuvempu University Shankaragatta, Shimoga Dst, Karnataka, India \\ ${ }^{3}$ Department of Organic Chemistry, Indian Institute of Science, Bangalore-560 012, Karnataka, India \\ ${ }^{4}$ Department of Biotechnology, Jnana Sahyadri Kuvempu University Shankaragatta, Shimoga Dst, Karnataka, India \\ ${ }^{5}$ Department of Biotechnology, M.S. Ramaiah Institute of Technology, Bangalore-560 054, Karnataka, India
}

\begin{abstract}
HCV (Hepatitis C virus) infection leads to complex liver diseases by affecting the hepatocytes. HCV Non-structural protein NS3 protease play major role in the processing of polyprotein and replication of HCV. NS5b is also a key enzyme in the replication of the virus. In our study we first selected 5 plants based on their significance in inhibiting liver disorders, viral infections and their use in treatment of viral infection. A library of 10 flavonoids were constructed and docked against NS3 protease and 5 sites of NS5B which includes active site and allosteric sites; palm site 1, palm site 2, thumb site 1, thumb site 2. quercetin 3-O-rutinoside showed the best interactions with both NS3 and 5 sites of NS5B. However, in-vitro studies have to be performed in order to confirm the inhibitory effect of these flavonoids.
\end{abstract}

Keywords: HCV, Antiviral, Flavonoids, Docking study, Protease and RdRp ****

\section{INTRODUCTION}

Hepatitis $\mathrm{C}$ virus (HCV) infection is one of the most complicated health conditions globally and it is estimated that around 180 million people are affected severely with this infection (Dubuisson J et al., 2014). HCV infects the host liver cells i.e. the hepatocytes and causes several liver diseases including advanced fibrosis, cirrhosis and hepatocellular carcinoma; it might even cause a reason for liver transplantation (Scheel $\mathrm{T}$ et al., 2013). Having this evident medical requirement, extensive efforts have been disbursed to develop effectual preventive and therapeutic strategies for hepatitis $\mathrm{C}$ infection (Bartenschlager $\mathrm{R}$ et al., 2013). Until 2011, the combination of pegylated interferon (PegIFN) $-\alpha$ and ribavirin was the most standard treatment for chronic hepatitis $\mathrm{C}$ infection having a success rate of 40$50 \%$ (Imran M et al., 2013). This treatment has various side effects including flu-like symptoms, anxiety and haemolytic anaemia and other contra-indications that are not advisable to patients from receiving the drugs. Moreover, antiviral therapy with interferon and ribavirin is very expensive. Therefore, more efficacious antiviral therapies are required.

Hepatitis C virus contains a single-stranded genomic RNA with a single open reading frame (ORF) that is necessary for the translation and replication of the viral genome. It encodes structural proteins including the core protein $\mathrm{C}$, two envelope glycoproteins E1 and E2 and viroporin p7; nonstructural proteins include NS2, NS3, NS4A, NS4B, NS5A and NS5B. Non-structural proteins are required for RNA replication (Calland $\mathrm{N}$ et al., 2012). NS5B is an RNA dependent RNA polymerase and it forms the key enzyme in the replication of the virus. On the other hand nonstructural protein NS3 protease directs proteolytic cleavages at the
NS2A/NS2B, NS2B/NS3, NS3/NS4A, NS4A/NS4B, and NS4B/NS5 junctions as well as at internal sites within $C$, NS2A, NS3, and NS4A. NS3/2B liberates the functional form of the viral polymerase and other viral proteins required for DENV replication. Thus, inhibitors of NS5b and NS3 may disrupt two separate processes relevant to the suppression of HCV infection.

The traditional and almost exclusive sources of active substances for most of the therapies are derived from plants. Currently, plants are the indirect or direct source of half of the approved drugs. Likewise, many herbs and isolated phyto-constituents have been investigated for inhibitory effect against HCV. Therefore plant based products such as flavonoids have been selected based on their known effects and its specificity and then it is screened to check the efficacy In vitro for toxicity. Flavonoids are basically a large group of polyphenolic compounds with a benzo- $\gamma$-pyrone structure and are ubiquitously present in most of the plants (Kumar S et al., 2013). Recent studies have demonstrated the inhibitory effect of flavonoids against HCV and Dengue viral replication. In view of this we investigated the antiviral efficacy of flavonoids by targeting HCV NS3 and NS5B enzymes.

\section{MATERIALS AND METHODS}

\subsection{Criteria for the Selection of Plants}

5 plants were selected for the study by using the following criteria: Plant extracts reported antiviral effect, Plants used by Traditional medical practitioners like Ayurveda and Chinese medicine to treat liver disorder and Plants reported to inhibit viral protease enzyme (ex HCV protease). 


\subsection{Selection of Compounds}

From the list of selected plants using the criteria mentioned above, the flavonoids that were reported in these plants were searched in online tools using search engines such as NCBI, $\mathrm{BMC}$, and Science Direct.

\subsection{Docking Studies}

Molecular docking of the flavonoids with NS3 protease and NS5B is done by the following steps:

\subsection{Preparation of Ligands}

The 2D structure of each flavonoid selected was drawn using Chem Draw software and was saved as a MOL file. These 2D structures were converted into 3D structures and optimized by minimizing their energy using Avogadro software and saved as a pdb file and were used as input file for opening in Auto Dock. The molecule was opened in Auto Dock software and the torsion was set in each molecule by making the number of rotatable bonds nil in them and was saved as a pdbqt file.

\subsection{Preparation of Receptor Protein}

The protein (NS3 protease) structure was downloaded from Protein Data Bank using PDB ID 1dy8A and 3TYVA for NS3 and NS5B respectively in pdb format. This was opened in Auto Dock and optimized by deleting the water molecule, removing the heteroatoms etc. and was saved as a pdbqt file. The grid box (having confirmation: center_x $=62.1$, center $y$ $=-12.092$, center $\mathrm{z}=8.457$ ) was chosen for the protein on their active site (His57, Ser139, Arg155, Ala157, Ala156).

\subsection{Molecular Docking}

Both the compound and the protein were docked using Autodock vina. The best ranked model with low binding energy was analyzed further and visualized using Ligplot software.

\section{RESULTS}

\subsection{Selection of Plants}

Based on the biological significance, 5 plants were selected which have already been used to treat several liver diseases, treat viral infections and inhibit viral proteases:

Table 1: Plants used in the study

\begin{tabular}{|l|l|l|}
\hline S1 no & Plants & References \\
\hline 1. & $\begin{array}{l}\text { Ricinus } \\
\text { communis }\end{array}$ & (Samvatsar and Diwanji 2000) \\
\hline 2. & $\begin{array}{l}\text { Nicotiana } \\
\text { glauca }\end{array}$ & (Janakat and Al-Merie 2002) \\
\hline 3. & $\begin{array}{l}\text { Phyllanthus } \\
\text { debilis }\end{array}$ & (Sane et al., 1995) \\
\hline 4. & $\begin{array}{l}\text { Phillyrea } \\
\text { latifolia }\end{array}$ & (Janakat and Al-Merie 2002) \\
\hline 5. & $\begin{array}{l}\text { Chelidonium } \\
\text { majus }\end{array}$ & (Monavari1 et al., 2012) \\
\hline
\end{tabular}

\subsection{Results of Docking Study}

Table 2: Flavonoids library

\begin{tabular}{|c|c|c|c|c|c|c|c|}
\hline \multirow[t]{2}{*}{ S1 no. } & \multirow[t]{2}{*}{ Flavonoids } & \multicolumn{6}{|c|}{ Binding affinity $(-\mathrm{kcal} / \mathrm{mol})$} \\
\hline & & NS3 & Active site & Palm 1 & Palm 2 & Thumb 1 & Thumb 2 \\
\hline 1 & Dihydrochelerythrine & 5.7 & 6.4 & 8.8 & 5.8 & 7.1 & 7.8 \\
\hline 2 & Epicatechin & 6.3 & 7.0 & 8.8 & 5.7 & 6.9 & 7.4 \\
\hline 3 & Fisetin & 6.3 & 7.3 & 8.6 & 5.7 & 6.5 & 7.5 \\
\hline 4 & Isoquercitrin & 7.6 & 8.2 & 11.5 & 7.4 & 8.5 & 8.8 \\
\hline 5 & $\begin{array}{l}\text { kaempferol-3-O- } \beta \text {-D- } \\
\text { glucopyranoside }\end{array}$ & 7.8 & 9.6 & 12.2 & 7.4 & 7.7 & 8.6 \\
\hline 6 & luteolin 4'-O-glucoside & 7.7 & 9.2 & 10.1 & 7.0 & 7.8 & 9.0 \\
\hline 7 & Patuletine & 6.6 & 6.9 & 9.6 & 5.9 & 6.8 & 7.6 \\
\hline 8 & $\begin{array}{l}\text { quercetin-3-O- } \beta \text {-D- } \\
\text { glucopyranoside }\end{array}$ & 8.0 & 8.4 & 10.4 & 7.0 & 7.3 & 8.0 \\
\hline 9 & quercetin 3-O-rutinoside & 8.1 & 9.3 & 9.9 & 7.8 & 8.5 & 9.1 \\
\hline 10 & quercetin xylopyranoside & 7.7 & 8.5 & 11.4 & 6.9 & 7.1 & 8.2 \\
\hline
\end{tabular}




\section{LIGPLOT ANALYSIS OF LEAD MOLECULES}

\subsection{Leads of NS5b}

Intaraction of kaempferol-3-O- $\beta$-D-glucopyranoside with NS5b active site

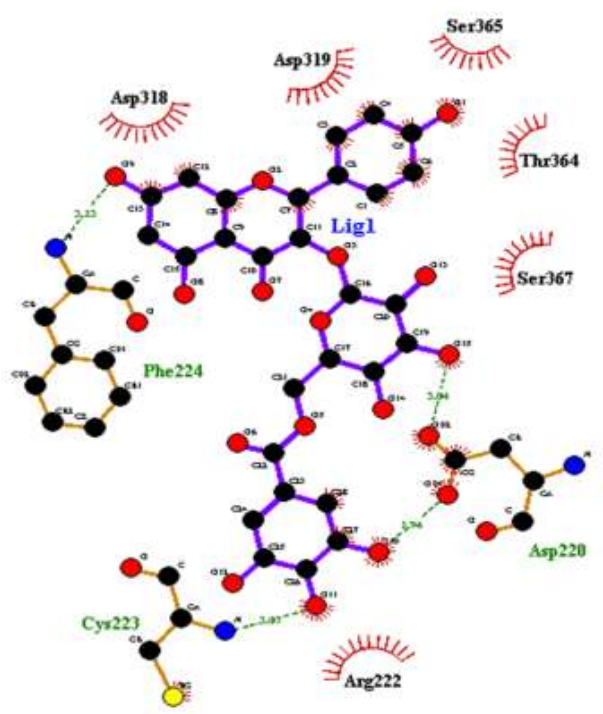

Hydrophilic interactions: Asp 220, Phe 224, cys 223; hydrophobic interactions: asp318, asp319, ser365, thr364, ser367, arg 222

Intaraction of quercetin 3-O- rutinoside with NS5b active site

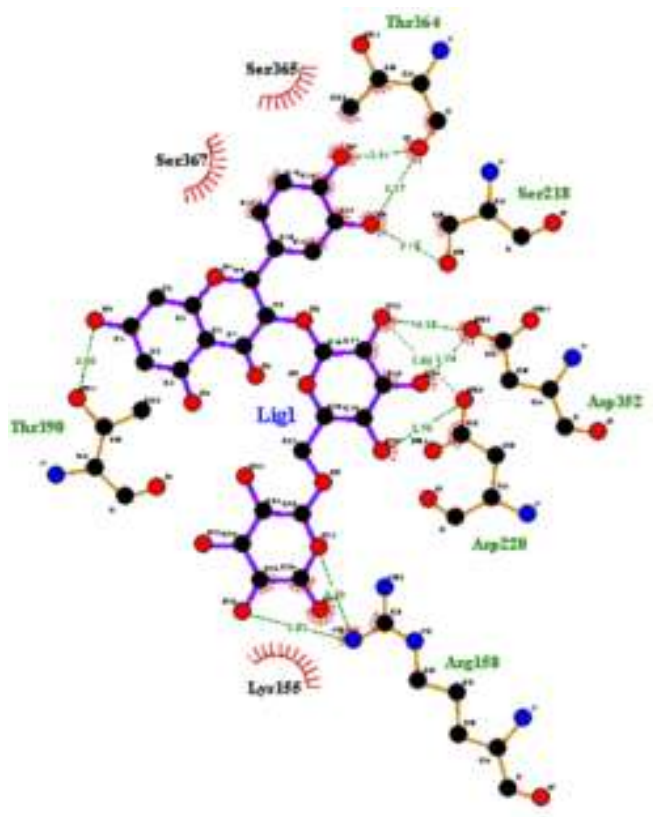

Hydrophilic interactions: Thr364, Ser 218, Asp 352, Asp 220, Arg 158, Thr 390; hydrophobic interactions: Ser 365, Ser 367, Lys 155

\subsection{Leads of NS3}

Intaraction of quercetin-3-O- $\beta$-D-glucopyranoside with NS3active sit

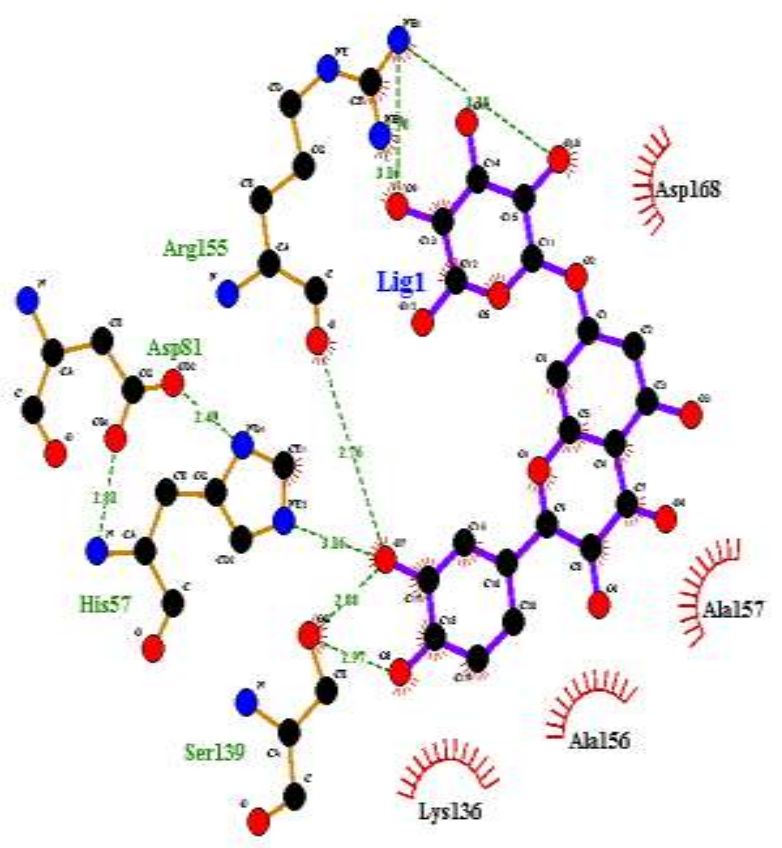

Hydrophilic interactions: arg155, asp81, his57, ser139; hydrophobic interactions: asp168, ala157, ala156, lys 136

Intaraction of quercetin 3-O- rutinoside with NS3active sit

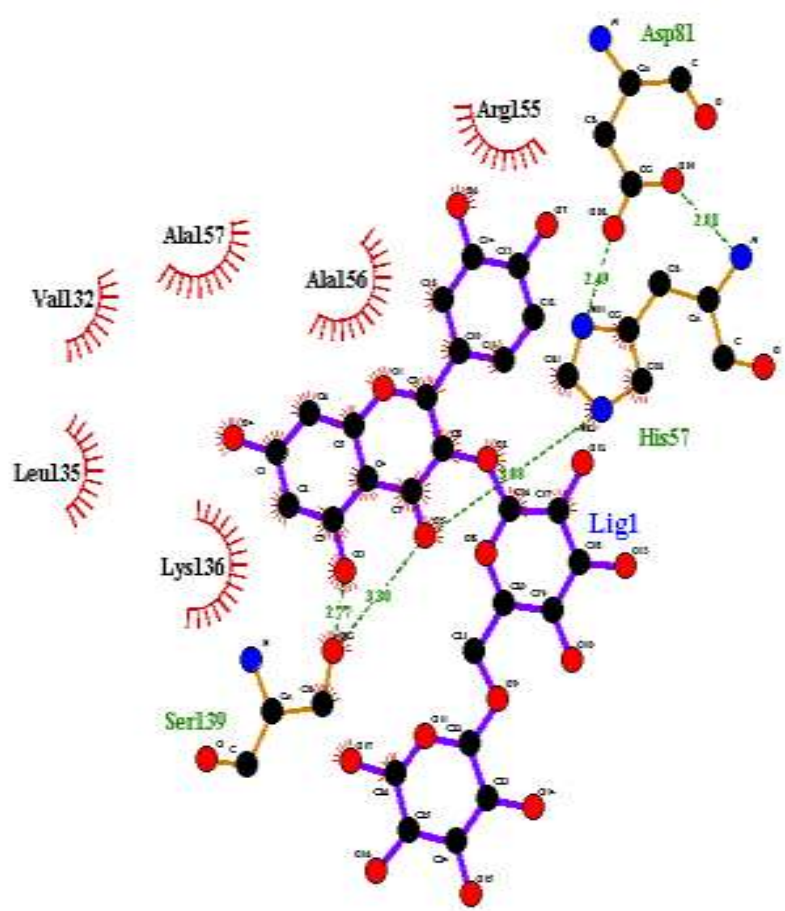

Hydrophilic interactions: asp81, his57, ser139; hydrophobic interactions: arg155, ala156, ala157, val132, leu135, lys 136 


\subsection{Leads of Palm 1}

Interaction of Isoquercitrin with NS5b Palm 1 site

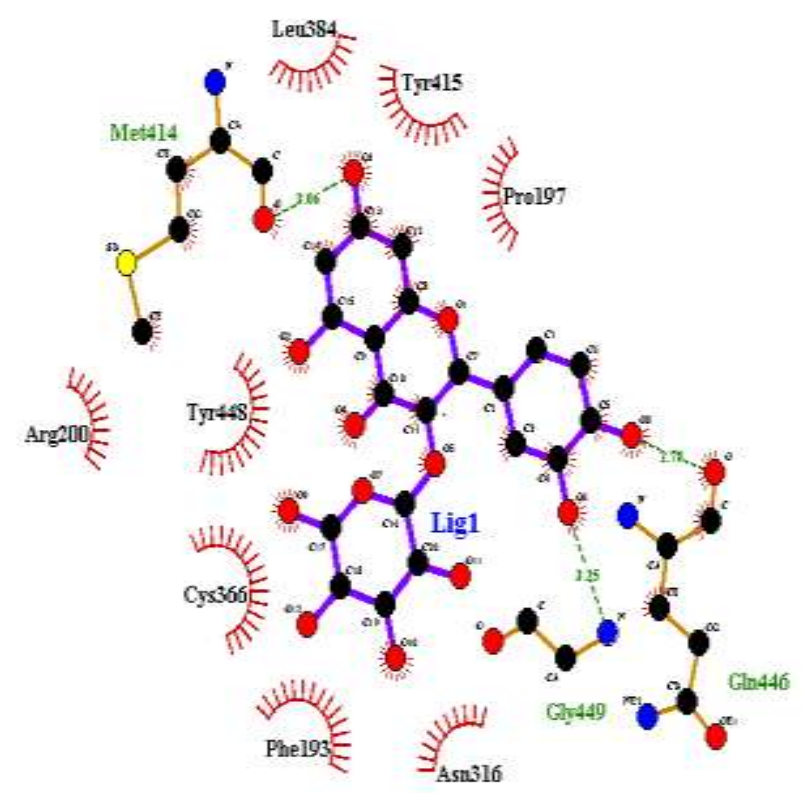

Hydrophilic interactions: met414, gly449, gln446; hydrophobic interactions: leu384, tyr415, pro197, arg200, tyr448, cys366, phe193, asn316

Interaction of kaempferol-3-O- $\beta$-D-glucopyranoside with NS5b Palm 1 site

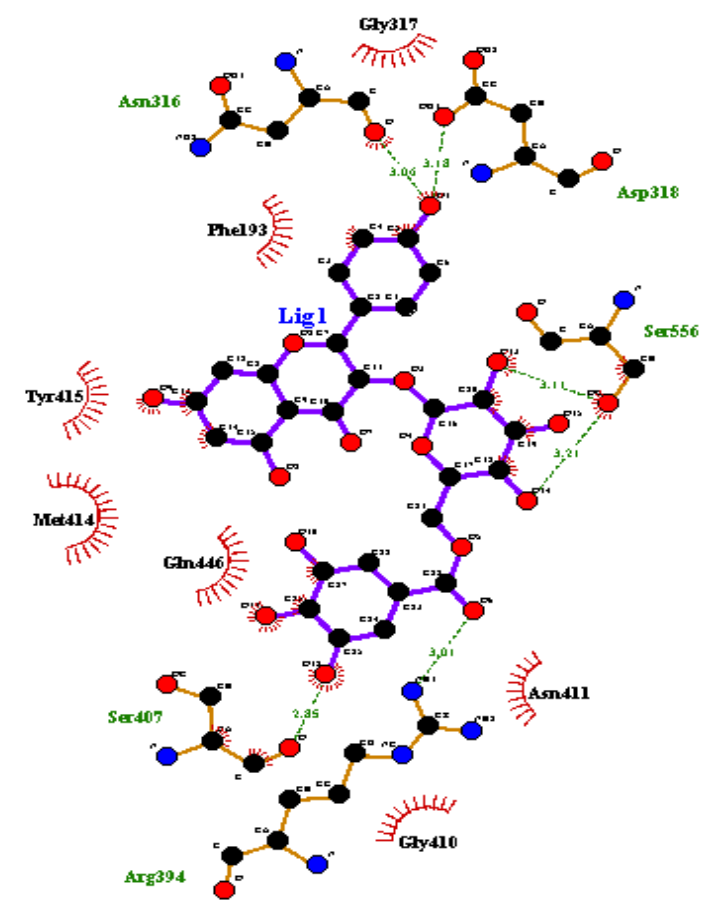

Hydrophilic interactions: asn316, asp 318, ser556, ser401, arg394; hydrophobic interactions: gly317, phe193, tyr 415, met414, gln446, asn411, gly410

\subsection{Leads of Palm 2}

Interaction of kaempferol-3-O- $\beta$-D-glucopyranoside with NS5b Palm 2 site

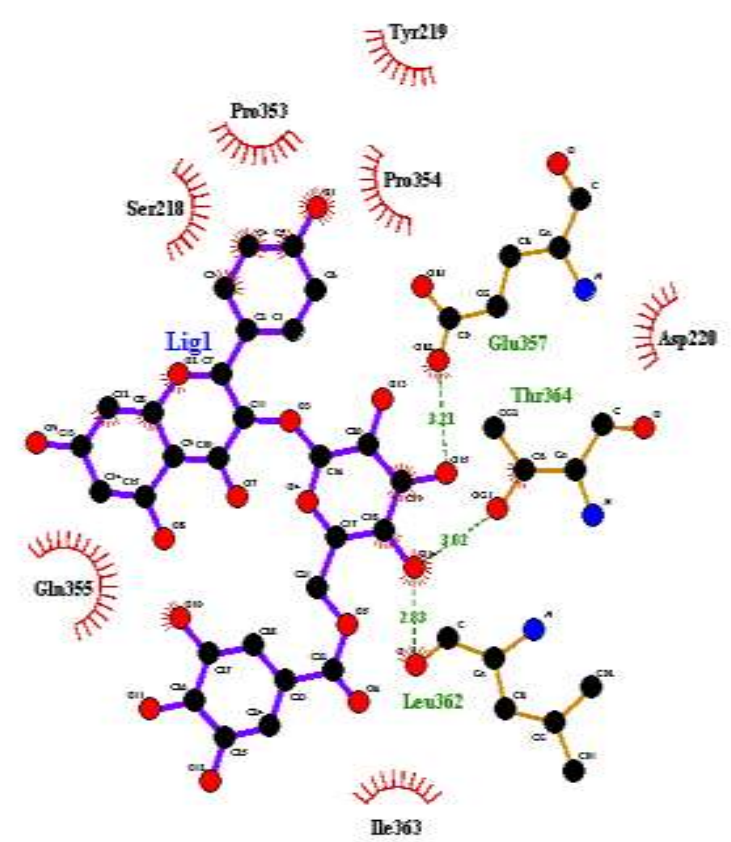

Hydrophilic interactions: glu357, thr 364, leu362; hydrophobic interactions: asp220, tyr219, pro354, pro363, ser218, gln355, ile 363

Interaction of quercetin 3-O-rutinoside with NS5b Palm 2 site

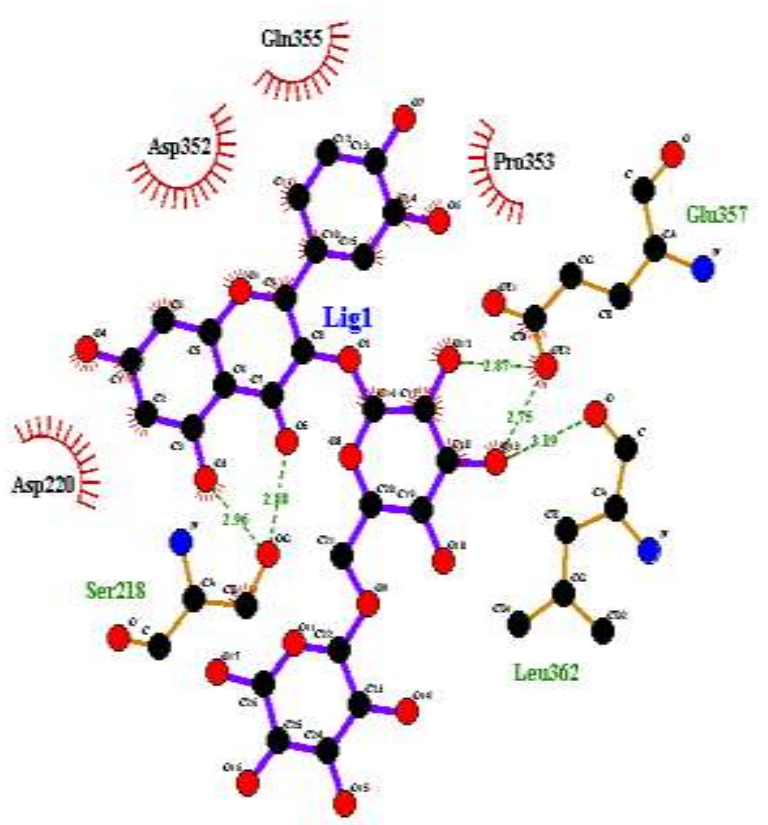

Hydrophilic interactions: ser218, leu362, glu357; hydrophobic interactions: asp352, gln355, pro353, asp220 


\subsection{Leads of Thumb 1}

Interaction of Isoquercitrin with NS5b Thumb 1 site

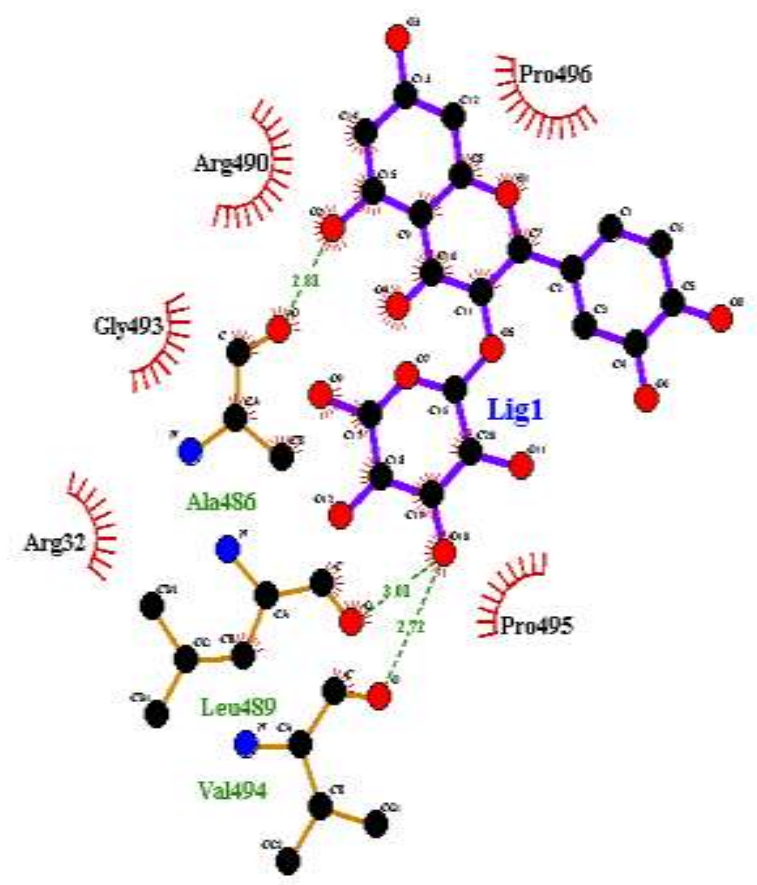

Hydrophilic interactions: ala486, leu489, val494; hydrophobic interactions: pro496, pro495, arg32, gly493, $\arg 490$

Interaction of quercetin 3-O-rutinoside with NS5b Thumb 1site

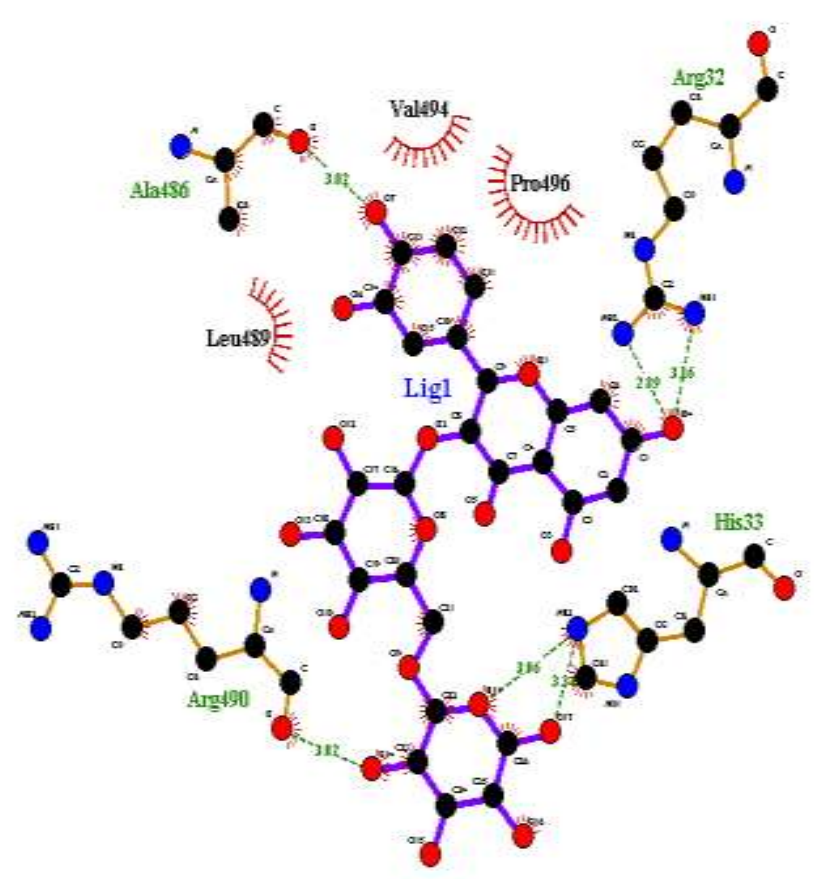

Hydrophilic interactions: ala486, arg32, his33, $\arg 490$; hydrophobic interactions: val 494, pro496, leu489

\subsection{Leads of Thumb 2}

Interaction of luteolin $4^{\prime}$-O-glucoside with NS5b Thumb 2 site

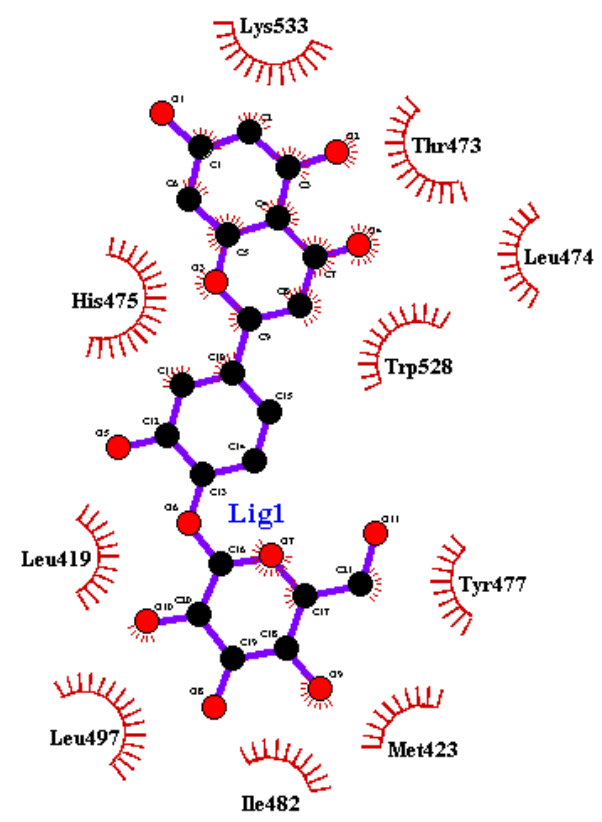

Hydrophobic interactions: lys 533, thr473, leu474, trp528, his 475 , leu419, leu497, ile482, met423, tyr477

Interaction of quercetin 3-O-rutinoside with NS5b Thumb 2 site

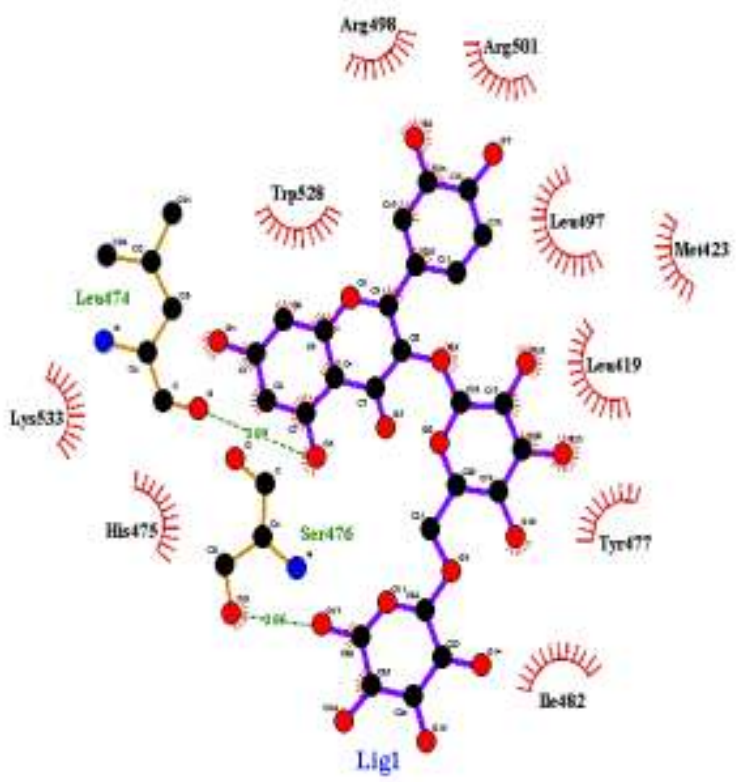

Hydrophilic interactions: leu474, ser476; hydrophobic interactions: lys533, his475, trp528, $\arg 498, \arg 501$, leu497, met423, leu419, tyr477, ile482

Fig 1: Interactions of lead molecule with grid box of enzymes 


\section{DISCUSSIONS}

Wide variety of medicinal plants has been extensively studied in terms of their anti-viral activity. Several hundreds of naturally active compounds have been recognized throughout the world. Mechanism of action is complementary and overlapping in most of them; it might either be by inhibiting replication of the virus or by obligating the synthesis of viral enzymes. These naturally active compounds offer a lot of circumstances for detecting novel structures that are active against a wide range of disease targets. In addition to this, they are capable of being absorbed and metabolized in the human system. The development costs of fabricating such orally active medicines are very economical. Therefore, natural products like flavonoids which is present in almost all plants offer great promise as potentially effective new antiviral drugs due to their simple structure and ease to manipulation for further design and synthesis.

In this study, first, 5 plants were selected based on their involvement in treating liver diseases, inhibiting viral proteases and treatment of viral infections (Table 1). These plants harbor a lot of natural compounds in them including terpenes, tannins, flavonoids and other phenolic which have immense medicinal properties such as being antiinflammatory, anti-ulcer and are used to treat several medical conditions such as cardiovascular, allergies, Alzheimer etc. There have been reports of these natural organic compounds used in the treatment of viral diseases such as HBV, HIV, Herpes simplex virus and HCV. Here we have concentrated mainly on the flavonoids that are present in these plants as they have a simpler structure when compared to other phenolic compounds and are also ubiquitously present in plants. A library of 10 flavonoids present in these 5 plants altogether was prepared (Table 2).

Molecular docking studies were performed with 10 flavonoids against the active site of NS3 protease, NS5B and the allosteric sites of NS5B. When the ligands and the protein is docked together, Autodock Vina searches for the best confirmation of the complex with least binding energy and hence the highest stability. In this way, it gives an output file of 9 best ranked models with different binding affinities in $\mathrm{kcal} / \mathrm{mol}$. The confirmation which is ranked $1 \mathrm{st}$ has the least binding affinity and is considered to be the best possible orientation that can be obtained in docking. For example docking of quercetin 3-O- rutinoside with NS3 protease: 1 to 9 models have the binding affinity of -8.1 , $7.8,-7.7,-7.6,-7.3,-7.3,-7.2,7.2,7.1 \mathrm{kcal} / \mathrm{mol}$ respectively. The model with the affinity $-8.1 \mathrm{kcal} / \mathrm{mol}$ will be selected for the further analysis. Similarly the 10 flavonoids were docked against the sites of NS5B which includes active site, palm site 1 , palm site 2 , thumb site 1 , thumb site 2 . Out of the 10 flavonoids quercetin 3-O- rutinoside showed the best interactions with both NS3 and 5 sites of NS5B having a binding affinity of $-8.1,-9.3,-9.9,-7.8,-8.5,-9.1$ with the same followed by kaempferol-3-O- $\beta$-D-glucopyranoside (figure 1).
When the best ranked model was merged with the pdb file of the protein and opened in the Ligplot analysis software, we get to observe different interactions of between each flavonoid and the amino acid residues in and around the active site of the protein. In each of the case, the ligand is represented purple in colour, the hydrophobic interactions are represented as a semi circled structure and the amino acids residues are in red, for the hydrophilic interactions the amino acid residues are represented in green. It even exhibits the bond length between the oxygen atoms. Quercetin 3-O-rutinoside being the best molecule among the ten exhibited Hydrophilic interactions: Thr364, Ser 218, Asp 352, Asp 220, Arg 158, Thr 390; hydrophobic interactions: Ser 365, Ser 367, Lys 155 with the active site of NS5B. It showed Hydrophilic interactions: asp81, his57, ser139; hydrophobic interactions: $\arg 155$, ala156, ala157, val132, leu135, lys136 with the NS3 protease. On the other hand hydrophilic interactions: ser218, leu362, glu357; hydrophobic interactions: asp352, gln355, pro353, asp220 were observed with palm 2 site of NS5B. It exhibited Hydrophilic interactions: ala486, arg32, his33, arg490; hydrophobic interactions: val 494, pro496, leu489 with thumb 1 site of NS5B and atlast it showed Hydrophilic interactions: leu474, ser476; hydrophobic interactions: lys533, his475, trp528, $\arg 498, \arg 501, \operatorname{leu} 497, \operatorname{met} 423$, leu419, tyr477, ile482 with thumb 2 site of NS5B. luteolin 4'-O-glucoside showed only Hydrophobic interactions: lys 533, thr473, leu474, trp528, his 475, leu419, leu497, ile482, met423, tyr477 with the thumb 2 site of NS5B (Figure 1).

Considering the lack of potent antiviral agents against HCV other than combinations of IFN- $\alpha$ with ribavirin that brings about potential antiviral effects, it is important to look out for the potential benefits of combination therapy with other new anti-HCV agents. Moreover, synergistic interactions could be expected if the two compounds have different sites of action. However in-vitro studies have to be performed to confirm the inhibition of the viral replication by these flavonoids.

\section{REFERENCES}

[1] Bartenschlager R, Lohmann V, Penin F (2013) The molecular and structural basis of advanced antiviral therapy for hepatitis C virus infection. Microbiology. 11: 482-496.

[2] Calland N, Dubuisson J, Rouillé Y, Seron K (2012) Hepatitis C Virus and Natural Compounds: New Antiviral Approach. Viruses. 4: 2197-2217.

[3] Dubuisson J, Cosset F (2014) Virology and cell biology of the hepatitis $\mathrm{C}$ virus life cycle. J Hepatol. 61: 3-13.

[4] Imran M, Manzoor S, Khattak NM, Khalid M, Ahmed QL, Parvaiz F, Tariq M, Ashraf J, Ashraf W, Azam S, Ashraf M (2013) Current and future therapies for hepatitis $\mathrm{C}$ virus infection: from viral proteins to host targets. Arch Virol. 10: 510-516.

[5] Janakat S, Al-Merie H (2002) Evaluation of hepatoprotective effect of Pistacia lentiscus, Phillyrea latifolia and Nicotiana glauca. J Ethnopharmacol. 83:135-138. 
[6] Kumar S, Pandey A (2013) Chemistry and Biological Activities of Flavonoids: An Overview. Scientific World J. 1: 1-13.

[7] Monavari1 SH, Shahrabadi MS, Keyvani H et al (2012) Evaluation of in vitro antiviral activity of Chelidonium majus L. against herpes simplex virus type-1. Afr J Microbiol Res. 6:4360-4364.

[8] Samvatsar S, Diwanji VB (2000) Plant sources for the treatment of jaundice in the tribals of Western Madhya Pradesh of India. $J$ Ethnopharmacol. 73:313-316.

[9] Sane RT, Kuber VV, Challisary MS et al (1995). Hepatoprotection by Phyllanthus amarus and Phyllanthus debilis in $\mathrm{CCl} 4$ induced liver dysfunction. Curr Sci. 68:1243-1246.

[10] Scheel T, Rice C (2013) Understanding the hepatitis $\mathrm{C}$ virus life cycle paves the way for highly effective therapies. Nat Med. 19: 837-849. 